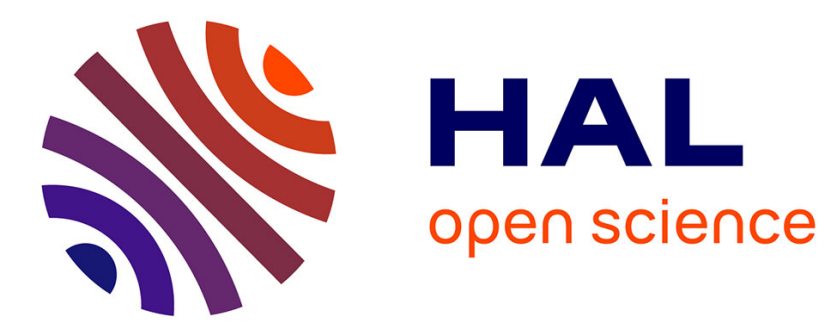

\title{
Diversification, convex preferences and non-empty core in the Choquet expected utility model
}

\author{
Alain Chateauneuf, Rose Anne Dana, Jean-Marc Tallon
}

\section{To cite this version:}

Alain Chateauneuf, Rose Anne Dana, Jean-Marc Tallon. Diversification, convex preferences and nonempty core in the Choquet expected utility model. Economic Theory, 2002, 19 (03), pp.509-523. halshs-00174770

\section{HAL Id: halshs-00174770 \\ https://shs.hal.science/halshs-00174770}

Submitted on 25 Sep 2007

HAL is a multi-disciplinary open access archive for the deposit and dissemination of scientific research documents, whether they are published or not. The documents may come from teaching and research institutions in France or abroad, or from public or private research centers.
L'archive ouverte pluridisciplinaire HAL, est destinée au dépôt et à la diffusion de documents scientifiques de niveau recherche, publiés ou non, émanant des établissements d'enseignement et de recherche français ou étrangers, des laboratoires publics ou privés. 


\title{
Optimal Risk-Sharing Rules and Equilibria with Choquet-Expected-Utility
}

\author{
Alain Chateauneuf, Rose-Anne Dana ${ }^{\dagger}$ Jean-Marc Tallon $\S^{\ddagger}$ \\ October 1997 \\ Revised July 1999
}

\begin{abstract}
This paper explores risk-sharing and equilibrium in a general equilibrium set-up wherein agents are non-additive expected utility maximizers. We show that when agents have the same convex capacity, the set of Pareto-optima is independent of it and identical to the set of optima of an economy in which agents are expected utility maximizers and have same probability. Hence, optimal allocations are comonotone. This enables us to study the equilibrium set. When agents have different capacities, matters are much more complex (as in the vNM case). We give a general characterization and show how it simplifies when Pareto-optima are comonotone. We use this result to characterize Pareto-optima when agents have capacities that are the convex transform of some probability distribution. comonotonicity of Paretooptima is also shown to be true in the two-state case if the intersection of the core of agents' capacities is non-empty; Pareto-optima may then be fully characterized in the two-agent, two-state case. This comonotonicity result does not generalize to more than two states as we show with a counter-example. Finally, if there is no-aggregate risk, we show that non-empty core intersection is enough to guarantee that optimal allocations are full-insurance allocation. This result does not require convexity of preferences.
\end{abstract}

Keywords: Choquet expected utility, comonotonicity, risk-sharing, equilibrium.

${ }^{*}$ CERMSEM, Université Paris I, 106-112 Bd de l'Hôpital, 75647 Paris Cedex 13, France. E-mail: chateaun@univ-paris1.fr

${ }^{\dagger}$ CEREMADE, Université Paris IX, Place du maréchal de Lattre de Tassigny, 75775 Paris Cedex 16, France. E-mail: dana@ceremade.dauphine.fr

${ }^{\ddagger}$ CNRS-EUREQua, 106-112 Bd de l’Hôpital, 75647 Paris Cedex 13, France. E-mail: jmtallon@univ-paris1.fr

${ }^{\S}$ We thank Sujoy Mukerji and an anonymous referee for useful comments. 


\section{Introduction}

In this paper, we explore the consequences of Choquet-expected-utility on risk-sharing and equilibrium in a general equilibrium set-up. There has been over the last fifteen years an extensive research on new decision-theoretic models (Karni and Schmeidler [1991] for a survey), and a large part of this research has been devoted to the Choquet-expected-utility model introduced by Schmeidler [1989]. However, applications to an economy-wide set-up have been relatively scarce. In this paper, we derive the implications of assuming such preference representation at the individuals level on the characteristics of Pareto optimal allocations. This, in turn, allows us to (partly) characterize equilibrium allocations under that assumption.

Choquet-expected-utility (CEU henceforth) is a model that deals with situations in which objective probabilities are not given and individuals are a priori not able to derive (additive) subjective probabilities over the state space. It is well-suited to represent agents' preferences in situation where "ambiguity" (as observed in the Ellsberg experiments) is a pervasive phenomenon $^{1}$. This model departs from expected-utility models in that it relaxes the sure-thing principle. Formally, the (subjective) expected-utility model is a particular subclass of the CEU of model. Our paper can then be seen as an exploration of how results established in the von NeumannMorgenstern (vNM henceforth) case are modified when allowing for more general preferences, whose form rests on sound axiomatic basis as well. Indeed, since CEU can be thought of as representing situations in which agents are faced with "ambiguous events", it is interesting to study how the optimal social risk-sharing rule in the economy is affected by this ambiguity and its perception by agents.

We focus on a pure-exchange economy in which agents are uncertain about future endowments and consume after uncertainty is resolved. Agents are CEU maximizers and characterized by a capacity and a utility index (assumed to be strictly concave).

When agents are vNM maximizers and have the same probability on the state space, it is well-known since Borch [1962] that agents' optimal consumptions depend only on aggregate risk, and is a non-decreasing function of aggregate resources: at an optimum, an agent bears only (some of) the aggregate risk. It is easy to fully characterize such Pareto Optima (see Eeck-

\footnotetext{
${ }^{1}$ See Schmeidler [1989], Ghirardato [1994], Mukerji [1997].
} 
houdt and Gollier [1995]). More generally, in the case of probabilized risk, Landsberger and Meilijson [1994] and Chateauneuf, Cohen and Kast [1997] have shown that Pareto Optima (P.O. henceforth) are comonotone if agents' preferences satisfy second-order stochastic dominance. This, in particular, is true in the rank-dependent-expected-utility case. The first goal of this paper is to provide a characterization of the set of P.O. and equilibria in the rank-dependent-expected-utility case. Our second and main aim is to assess whether the results obtained in the case of risk are robust when one moves to a situation of non-probabilized uncertainty with Choquet-expected-utility, in which there is some consensus.

We first study the case where all agents have the same capacity. We show that if this capacity is convex, the set of P.O. is the same as that of an economy with vNM agents whose beliefs are described by a common probability. Furthermore, it is independent of that capacity. As a consequence, P.O. are easily characterized in this set-up, and depend only on aggregate risk (and utility index). Thus, if uncertainty is perceived by all agents in the same way, the optimal risk-bearing is not affected (compared to the standard vNM case) by this ambiguity. The equivalence proof relies heavily on the fact that, if agents are vNM maximizers with identical beliefs, optimal allocations are comonotone and independent of these beliefs : each agent's consumption moves in the same direction as aggregate endowments. This equivalence result is in the line of a result on aggregation in appendix $\mathrm{C}$ of Epstein and Wang [1994]. Finally, the information given by the optimality analysis is used to study the equilibrium set. A qualitative analysis of the equilibrium correspondence may be found in Dana [1998].

When agents have different capacities, matters are much more complex. To begin with, in the vNM case, we don't know of any conditions ensuring that P.O. are comonotone in that case. However, in the CEU model, intuition might suggest that if agents have capacities whose cores have some probability distribution in common, P.O. are then comonotone. This intuition is unfortunately not correct in general, as we show with a counterexample. As a result, when agents have different capacities, whether P.O. allocations are comonotone depends on the specific characteristics of the economy. On the other hand, if P.O. are comonotone, they can be further characterized, although not fully. It is also in general non-trivial to use that information to infer properties of equilibrium. This leads us to study cases 
for which it is possible to prove that P.O. allocations are comonotone.

A first case is when the agents' capacities are the convex transform of some probability distribution. We then know from Chateauneuf, Cohen and Kast [1997] and Landsberger and Meilijson [1994] that P.O. are comonotone. Our analysis then enables us to be more specific than they are about the optimal risk-bearing arrangements and equilibrium of such an economy.

The second case is the simple case in which there are only two states (as in simple insurance models $\grave{a}$ la Mossin [1968]). The non-emptiness of the cores' intersection is then enough to prove that P.O. allocations are comonotone, although it is not clear what the actual optimal risk-sharing arrangement looks like. If we specify the model further and assume there are only two agents, the risk-sharing arrangement can be fully characterized. Depending on the specifics of the agents' characteristics, it is either a subset of the P.O. of the economy in which agents each have the probability that minimizes, among the probability distributions in the core, the expected value of aggregate endowments, or the less pessimistic agent insures the other. (This last risk-sharing arrangement typically cannot occur in a vNM setup with different beliefs and strictly concave utility functions.) The equilibrium allocation in this economy can also be characterized.

Finally, we consider the situation in which there exists only individual risk, a case first studied by Malinvaud [1972] and [1973]. comonotonicity is then equivalent to full-insurance. We show that a condition for optimal allocations to be full-insurance allocations is that the intersection of the core of the agents' capacities is non-empty, a condition that can be intuitively interpreted as minimum consensus. This full-insurance result easily generalizes to the multi-dimensional set-up. Using this result, we show that establish that any equilibrium of particular vNM economies is an equilibrium of the CEU economy. These vNM economies are those in which agents have the same characteristics as in the CEU economy and have common beliefs given by a probability in the intersection of the cores of the capacities of the CEU economy. When the capacities are convex, any equilibrium of the CEU economy is of that type. This equivalence result between equilibrium of the CEU economy and associated vNM economies suggests that equilibrium is indeterminate, an idea further explored in Tallon [1997] and Dana [1998].

The rest of the paper is organized as follows. Section 2 establishes notation and define the characteristics of the pure exchange economy that we 
deal with in the rest of the paper. In particular, we recall properties of the Choquet integral. We also recall there some useful information on optimal risk-sharing in vNM economies. Section 3 is the heart of the paper and deals with the general case of convex capacities. In a first sub-section, we assume that agents have identical capacities, while the second sub-section deals with the case where agents have different capacities. Section 4 is devoted to the study of two particular cases of interest, namely the case where agents' capacities are the convex transform of a common probability distribution and the two-state case. The case of no-aggregate risk in a multi-dimensional set-up is studied in section 5 .

\section{Notation, definitions and useful results}

We consider an economy in which agents make decisions before uncertainty is resolved. The economy is a standard two-period pure-exchange economy, but for agents' preferences.

There are $k$ possible states of the world, indexed by superscript $j$. Let $S$ be the set of states of the world and $\mathcal{A}$ the set of subsets of $S$. There are $n$ agents indexed by subscript $i$. We assume there is only one $\operatorname{good}^{2}$. $C_{i}^{j}$ is the consumption by agent $i$ in state $j$ and $C_{i}=\left(C_{i}^{1}, \ldots, C_{i}^{k}\right)$. Initial endowments are denoted $w_{i}=\left(w_{i}^{1}, \ldots, w_{i}^{k}\right) . w=\sum_{i=1}^{n} w_{i}$ is the aggregate endowment.

We will focus on Choquet-Expected-Utility. We assume the existence of a utility index $U_{i}: \mathbb{R}_{+} \rightarrow \mathbb{R}$ that is cardinal, i.e. defined up to a positive affine transformation. Throughout the paper $U_{i}$ is taken to be strictly increasing and strictly concave. When needed, we will assume differentiability together with the usual Inada condition:

Assumption U1: $\forall i, U_{i}$ is $C^{1}$ and $U_{i}^{\prime}(0)=\infty$.

Before defining CEU (the Choquet integral of $U$ with respect to a capacity), we recall some properties of capacities and their core.

\footnotetext{
${ }^{2}$ In section 5 , we will deal with several goods and will introduce the appropriate notation at that time.
} 


\subsection{Capacities and the core}

A capacity is a set function $\nu: \mathcal{A} \rightarrow[0,1]$ such that $\nu(\emptyset)=0, \nu(S)=1$, and, for all $A, B \in \mathcal{A}, A \subset B \Rightarrow \nu(A) \leq \nu(B)$. We will assume throughout that the capacities we deal with are such that $1>\nu(A)>0$ for all $A \in \mathcal{A}$, $A \neq S, A \neq \emptyset$.

A capacity $\nu$ is convex if for all $A, B \in \mathcal{A}, \nu(A \cup B)+\nu(A \cap B) \geq$ $\nu(A)+\nu(B)$.

The core of a capacity $\nu$ is defined as follows

$$
\operatorname{core}(\nu)=\left\{\pi \in \mathbb{R}_{+}^{k} \mid \sum_{j} \pi^{j}=1 \text { and } \pi(A) \geq \nu(A), \forall A \in \mathcal{A}\right\}
$$

where $\pi(A)=\sum_{j \in A} \pi^{j}$. Core $(\nu)$ is a compact, convex set which may be empty. Since $1>\nu(A)>0 \forall A \in \mathcal{A}, A \neq S, A \neq \emptyset$, any $\pi \in \operatorname{core}(\nu)$ is such that $\pi \gg 0$, (i.e., $\pi^{j}>0$ for all $j$ ).

It is well-known that when $\nu$ is convex, its core is non-empty. It is equally well-known that non-emptiness of the core does not require convexity of the capacity. If there are only two states however, it is easy to show that $\operatorname{core}(\nu) \neq \emptyset$ if and only if $\nu$ is convex.

We shall provide an alternative definition of the core in the following sub-section.

\subsection{Choquet-expected-utility}

We now turn to the definition of the Choquet integral of $f \in \mathbb{R}^{S}$ :

$$
\int f d \nu \equiv E_{\nu}(f)=\int_{-\infty}^{0}(\nu(f \geq t)-1) d t+\int_{0}^{\infty} \nu(f \geq t) d t
$$

Hence, if $f^{j}=f(j)$ is such that $f^{1} \leq f^{2} \leq \ldots \leq f^{k}$ :

$$
\int f d \nu=\sum_{j=1}^{k-1}[\nu(\{j, \ldots, k\})-\nu(\{j+1, \ldots, k\})] f^{j}+\nu(\{k\}) f^{k}
$$

As a consequence, if we assume that an agent consumes $C^{j}$ in state $j$, and that $C^{1} \leq \ldots \leq C^{k}$, then his preferences are represented by:

$v(C)=[1-\nu(\{2, . ., k\})] U\left(C^{1}\right)+\ldots[\nu(\{j, . ., k\})-\nu(\{j+1, . ., k\})] U\left(C^{j}\right)+\ldots \nu(\{k\}) U\left(C^{k}\right)$

Observe that, if we keep the same ranking of the states as above, then $v(C)=E_{\pi} U(C)$, where $C$ is here the random variable giving $C^{j}$ in state $j$, 
and the probability $\pi$ is defined by: $\pi^{j}=\nu(\{j, \ldots, k\})-\nu(\{j+1, \ldots, k\})$, $j=1, \ldots, k-1$ and $\pi^{k}=\nu(\{k\})$.

If $U$ is differentiable and $\nu$ is convex, the function $v: \mathbb{R}_{+}^{k} \rightarrow \mathbb{R}$ defined above is continuous, strictly concave and subdifferentiable. Let $\partial v(C)=$ $\left\{a \in \mathbb{R}^{k} \mid v(C)-v\left(C^{\prime}\right) \geq a\left(C-C^{\prime}\right), \forall C^{\prime} \in \mathbb{R}_{+}^{k}\right\}$ denote the subgradient of the function $v$ at $C$. In the open set $\left\{C \in \mathbb{R}_{+}^{k} \mid 0<C^{1}<C^{2}<\ldots<C^{k}\right\}$, $v$ is differentiable. If $0<C^{1}=C^{2}=\ldots=C^{k}$ then, $\partial v(C)$ is proportional to $\operatorname{core}(\nu)$.

The following proposition gives an alternative representation of $\operatorname{core}(\nu)$ that will be useful in section 5 .

Proposition $2.1 \operatorname{core}(\nu)=\left\{\pi \in \mathbb{R}_{+}^{k} \mid \sum_{j=1}^{k} \pi^{j}=1\right.$ and $\left.v(C) \leq E_{\pi}(U(C)), \forall C \in \mathbb{R}_{+}^{k}\right\}$

Proof: Let $\pi \in \operatorname{core}(\nu)$ and assume $C^{1} \leq C^{2} \leq \ldots \leq C^{k}$. Then,

$v(C)=U\left(C^{1}\right)+\nu(\{2, \ldots, k\})\left(U\left(C^{2}\right)-U\left(C^{1}\right)\right)+\ldots+\nu(\{k\})\left(U\left(C^{k}\right)-U\left(C^{k-1}\right)\right)$

Hence, since $\pi \in \operatorname{core}(\nu)$, and therefore $\nu(A) \leq \pi(A)$ for all events $A$ :

$v(C) \leq U\left(C^{1}\right)+\sum_{j=2}^{k} \pi^{j}\left(U\left(C^{2}\right)-U\left(C^{1}\right)\right)+\ldots+\pi^{k}\left(U\left(C^{k}\right)-U\left(C^{k-1}\right)\right)=E_{\pi}(U(C))$

which proves one inclusion.

To prove the other inclusion, let $\pi \in\left\{\pi \in \mathbb{R}_{+}^{k} \mid \sum_{j=1}^{k} \pi^{j}=1, v(C) \leq E_{\pi}(U(C)), \forall C\right\}$.

Normalize $U$ so that $U(\underline{C})=0$ and $U(\bar{C})=1$ for some $\underline{C}$ and $\bar{C}$. Let $A \in \mathcal{A}$ and $C^{A}=\underline{C} \mathbf{1}_{A^{c}}+\bar{C} \mathbf{1}_{A}$. Since $v\left(C^{A}\right)=\nu(A) \leq E_{\pi}\left(U\left(C^{A}\right)\right)=\pi(A)$, one gets $\pi \in \operatorname{core}(\nu)$.

A corollary is that if core $(\nu) \neq \emptyset$, then $v(C) \leq \min _{\pi \in \operatorname{core}(\nu)} E_{\pi} U(C) .{ }^{3}$

\section{3 comonotonicity}

We finally define comonotonicity of a class of random variables $\left(\widetilde{C}_{i}\right)_{i=1, \ldots, n}$. This notion, which has a natural interpretation in terms of mutualization of risks, will be crucial in the rest of the analysis.

Definition 1 A family $\left(\widetilde{C}_{i}\right)_{i=1, \ldots, n}$ of random variables on $S$ is a class of comonotone functions if for all $i, i^{\prime}$, and for all $j, j^{\prime},\left[C_{i}^{j}-C_{i}^{j^{\prime}}\right]\left[C_{i^{\prime}}^{j}-C_{i^{\prime}}^{j^{\prime}}\right] \geq$ 0 .

\footnotetext{
${ }^{3}$ It is well-known (see Schmeidler [1986]) that when $\nu$ is convex, the Choquet integral of any random variable $f$ is given by $\int f d \nu=\min _{\pi \in \operatorname{core}(\nu)} E_{\pi} f$.
} 
An alternative characterization is given in the following proposition (see Denneberg [1994]):

Proposition 2.2 A family $\left(\widetilde{C}_{i}\right)_{i=1, \ldots, n}$ of non-negative random variables on $S$ is a class of comonotone functions if and only if for all $i$, there exists a function $g_{i}: \mathbb{R}_{+} \rightarrow \mathbb{R}_{+}$, non-decreasing and continuous, such that for all $x \in \mathbb{R}_{+}, \sum_{i=1}^{n} g_{i}(x)=x$ and $C_{i}^{j}=g_{i}\left(\sum_{m=1}^{n} C_{m}^{j}\right)$ for all $j$.

The family $\left(\widetilde{C}_{i}\right)_{i=1, \ldots, n}$ is comonotone if they all vary in the same direction as their sum.

\subsection{Optimal risk-sharing with vNM agents}

We briefly recall here some well-known results on optimal risk-sharing in the traditional vNM case (see e.g. Eeckhoudt and Gollier [1995] or Magill and Quinzii [1996]). Consider first the case of identical vNM beliefs. Agents have the same probability $\pi=\left(\pi^{1}, \ldots, \pi^{k}\right), \pi^{j}>0$ for all $j$, over the states of the world and a utility function defined by $v_{i}\left(C_{i}\right)=\sum_{j=1}^{k} \pi^{j} U_{i}\left(C_{i}^{j}\right), i=$ $1, \ldots, n$. The following proposition recalls that the P.O. allocations of this economy are independent of the (common) probability, depend only on aggregate risk (and utility indices), and are comonotone ${ }^{4}$.

Proposition 2.3 Let $\left(C_{i}\right)_{i=1}^{n} \in \mathbb{R}_{+}^{k n}$ be a P.O. allocation of an economy in which agents have vNM utility index and identical additive beliefs $\pi$. Then, it is a P.O. of an economy with additive beliefs $\pi^{\prime}$ (and same vNM utility index). Furthermore, $\left(C_{i}\right)_{i=1}^{n}$ is comonotone.

As a consequence of propositions 2.2 and 2.3 , it is easily seen that, at a P.O. allocation, agent $i$ 's consumption $C_{i}$ is a non-decreasing function of $w$.

If agents have different probabilities $\pi_{i}^{j}, j=1, \ldots, k, i=1, \ldots, n$, over the states of the world, it is easily seen that P.O. now depend on the probabilities and on aggregate risk. It is actually easy to find examples in which P.O. are not necessarily comonotone (take for instance a model without aggregate risk in which agents have different beliefs: the P.O. allocations are not state-independent and therefore are not comonotone).

${ }^{4}$ Borch [1962] noted that, in a reinsurance market, at a P.O., "the amount which company $i$ has to pay will depend only on (...) the total amount of claims made against the industry. Hence any Pareto optimal set of treaties is equivalent to a pool arrangement." Note that this corresponds to the characterization of comonotone variables as stated in proposition 2.2. 


\section{Optimal risk-sharing and equilibrium with CEU agents: the general convex case}

In this section we deal first with optimal risk-sharing and equilibrium analysis when agents have identical convex capacities and then move on to different convex capacities.

\subsection{Optimal risk-sharing and equilibrium with identical ca- pacities}

Assume here that all agents have the same capacity $\nu$ over the states of the world and that this capacity is convex. We denote by $\mathcal{E}_{1}$ the exchange economy in which agents are CEU with capacity $\nu$ and utility index $U_{i}, i=$ $1, \ldots, n$.

Define $D_{\nu}(w)$ as follows:

$$
D_{\nu}(w)=\left\{\pi \in \operatorname{core}(\nu) \mid E_{\pi} w=E_{\nu} w\right\}
$$

The set $D_{\nu}(w)$ is constituted of the probabilities that "minimize the expected value of the aggregate endowment". In particular, if $w^{1}<w^{2} \ldots<$ $w^{k}, D_{\nu}(w)$ contains only $\pi=\left(\pi^{1}, \ldots, \pi^{k}\right)$ with $\pi^{j}=\nu(\{j, j+1, \ldots, k\})-$ $\nu(\{j+1, \ldots, k\})$ for all $j<k$ and $\pi^{k}=\nu(\{k\})$. If $w^{1}=\ldots=w^{k}$, the set $D_{\nu}(w)$ is equal to core $(\nu)$.

It is important to note that the Choquet integral of any random variable that is non-decreasing with $w$ is actually the integral of that random variable with respect to a probability distribution in $D_{\nu}(w)$. In particular, we have the following lemma.

Lemma 3.1 Let $\nu$ be a convex capacity, $U$ an increasing function and $C \in$ $\mathbb{R}_{+}^{k}$ be a non-decreasing function of $w$. Then, $v(C)=E_{\pi} U(C)$ for any $\pi \in D_{\nu}(w)$.

Proof: Since $C$ is non-decreasing in $w$, if $w^{1} \leq \ldots \leq w^{k}$, then $C^{1} \leq \ldots \leq$ $C^{k}$. Furthermore, $w^{j}=w^{j^{\prime}}$ implies $C^{j}=C^{j^{\prime}}$. The same relationship holds between $\left(w^{j}\right)_{j=1}^{k}$ and $\left(U\left(C^{j}\right)\right)_{j=1}^{k}, U$ being increasing. It is then simply a matter of writing down the expression of the Choquet integral to see that $v(C)=E_{\pi} U(C)$ for any $\pi \in D_{\nu}(w)$.

Proposition 3.1 The allocation $\left(C_{i}\right)_{i=1}^{n} \in \mathbb{R}_{+}^{k n}$ is a P.O. of $\mathcal{E}_{1}$ if and only if it is a P.O. of an economy in which agents have vNM utility index $U_{i}, i=$ 
$1, \ldots, n$ and identical probability over the set of states of the world. In particular, P.O. are comonotone.

Proof: Since the P.O. of an economy with vNM agents with same probability are independent of the probability, we can choose w.l.o.g. this probability to be $\pi \in D_{\nu}(w)$.

Let $\left(C_{i}\right)_{i=1}^{n}$ be a P.O. of the vNM economy. Being a P.O., this allocation is comonotone. By proposition 2.2, $C_{i}$ is a non-decreasing function of $w$. Hence, applying lemma 3.1, $v_{i}\left(C_{i}\right)=E_{\pi}\left[U_{i}\left(C_{i}\right)\right], i=1, \ldots, n$. If it were not a P.O. of $\mathcal{E}_{1}$, there would exist an allocation $\left(C_{1}^{\prime}, C_{2}^{\prime} \ldots C_{n}^{\prime}\right)$ such that

$$
v_{i}\left(C_{i}^{\prime}\right)=E_{\nu}\left[U_{i}\left(C_{i}^{\prime}\right)\right] \geq v_{i}\left(C_{i}\right)=E_{\pi}\left[U_{i}\left(C_{i}\right)\right]
$$

for all $i$, and with at least one strict inequality. Since $E_{\pi}\left[U_{i}\left(C_{i}^{\prime}\right)\right] \geq E_{\nu}\left[U_{i}\left(C_{i}^{\prime}\right)\right]$ for all $i$, this contradicts the fact that $\left(C_{i}\right)_{i=1}^{n}$ is a P.O. of the vNM economy.

Let $\left(C_{i}\right)_{i=1}^{n}$ be a P.O. of $\mathcal{E}_{1}$. If it were not a P.O. of the economy with vNM agents with probability $\pi$, there would exist a P.O. $\left(C_{i}^{\prime}\right)_{i=1}^{n}$ such that $E_{\pi}\left[U_{i}\left(C_{i}^{\prime}\right)\right] \geq E_{\pi}\left[U_{i}\left(C_{i}\right)\right] \geq v_{i}\left(C_{i}\right)$ for all $i$, and with a strict inequality for at least an agent. $\left(C_{i}^{\prime}\right)_{i=1}^{n}$ being Pareto optimal, it is comonotone and it follows by proposition 2.2 that $C_{i}^{\prime}$ is a non-decreasing function of $w$. Hence, applying lemma $3.1, v_{i}\left(C_{i}^{\prime}\right)=E_{\pi}\left[U_{i}\left(C_{i}^{\prime}\right)\right], i=1, \ldots, n$. This contradicts the fact that $\left(C_{i}\right)_{i=1}^{n}$ is a P.O. of $\mathcal{E}_{1}$.

Note that this proposition not only shows that P.O. allocations are comonotone in the CEU economy, but also completely characterizes them.

We may now also fully characterize the equilibria of $\mathcal{E}_{1}$.

\section{Proposition 3.2}

(i) Let $\left(p^{\star}, C^{\star}\right)$ be an equilibrium of a vNM economy in which all agents have utility index $U_{i}$ and beliefs given by $\pi \in D_{\nu}(w)$, then $\left(p^{\star}, C^{\star}\right)$ is an equilibrium of $\mathcal{E}_{1}$.

(ii) Conversely, assume U1. If $\left(p^{\star}, C^{\star}\right)$ is an equilibrium of $\mathcal{E}_{1}$, then there exists $\pi \in D_{\nu}(w)$ such that $\left(p^{\star}, C^{\star}\right)$ is an equilibrium of the vNM economy with utility index $U_{i}$ and probability $\pi \in D_{\nu}(w)$.

Proof: See Dana [1998]. 
Corollary 3.1 If $\mathbf{U} 1$ is fulfilled and $w^{1}<w^{2}<\ldots<w^{k}$, then the equilibria of $\mathcal{E}_{1}$ are identical to those of a vNM economy in which agents have utility index $U_{i}, i=1, \ldots, n$ and same probabilities over states $\pi^{j}=$ $\nu\{j, j+1, \ldots, k\}-\nu\{j+1, \ldots, k\}, j<k$ and $\pi^{k}=\nu(\{k\})$. Hence, $\left(w, C_{i}^{\star}, i=1, \ldots, n\right)$ are comonotone.

To conclude this sub-section, observe that P.O. allocations in the CEU economy inherits all the nice properties of P.O. allocations in a vNM economy with identical beliefs. In particular, P.O. allocations are independent of the capacity. However, the equilibrium allocations in the vNM economy do depend on beliefs, and it is not trivial to assess the relationship between the equilibrium set of a vNM economy with identical beliefs and the equilibrium set of the CEU economy $\mathcal{E}_{1}$. Note for instance that $\mathcal{E}_{1}$ has "as many equilibria" as there are probability distributions in the set $D_{\nu}(w)$. If $D_{\nu}(w)$ consists of a unique probability distribution, equilibria of $\mathcal{E}_{1}$ are the equilibria of the vNM economy with beliefs equal to that probability distribution. On the other hand, if $D_{\nu}(w)$ is not a singleton, it is a priori not possible to assimilate all the equilibria of $\mathcal{E}_{1}$ with equilibria of a given vNM economy.

\subsection{Optimal risk-sharing and equilibrium with different ca- pacities}

We next consider an economy in which agents have different convex capacities. Denote the economy in which agents are CEU with capacity $\nu_{i}$ and utility index $U_{i}, i=1, \ldots, n$ by $\mathcal{E}_{2}$.

We first give a general characterization of the set of P.O., when no further restrictions are imposed on the economy. We then show that this general characterization can be most usefully applied when one knows that P.O. are comonotone.

Proposition 3.3 $(i)$ Let $\left(C_{i}\right)_{i=1}^{n} \in \mathbb{R}_{+}^{k n}$ be a P.O. of $\mathcal{E}_{2}$ such that for all $i$, $C_{i}^{j} \neq C_{i}^{\ell}, j \neq \ell$. Let $\pi_{i} \in \operatorname{core}\left(\nu_{i}\right)$ be such that $E_{\nu_{i}}\left[U_{i}\left(C_{i}\right)\right]=E_{\pi_{i}}\left[U_{i}\left(C_{i}\right)\right]$ for all $i$. Then $\left(C_{i}\right)_{i=1}^{n}$ is a P.O. of an economy in which agents have vNM utility index $U_{i}$ and probabilities $\pi_{i}, i=1, \ldots, n$.

(ii) Let $\pi_{i} \in \operatorname{core}\left(\nu_{i}\right), i=1, \ldots, n$ and $\left(C_{i}\right)_{i=1}^{n}$ be a P.O. of the vNM economy with utility index $U_{i}$ and probabilities $\pi_{i}, i=1, \ldots, n$. If $E_{\nu_{i}}\left[U_{i}\left(C_{i}\right)\right]=$ $E_{\pi_{i}}\left[U_{i}\left(C_{i}\right)\right]$ for all $i$, then $\left(C_{i}\right)_{i=1}^{n}$ is a P.O. of $\mathcal{E}_{2}$. 
Proof: $(i)$ If $\left(C_{i}\right)_{i=1}^{n}$ is not a P.O. of a vNM economy, then there exists $\left(C_{i}^{\prime}\right)_{i=1}^{n}$ such that $E_{\pi_{i}}\left[U_{i}\left(C_{i}^{\prime}\right)\right] \geq E_{\nu_{i}}\left[U_{i}\left(C_{i}\right)\right]$ with a strict inequality for some $i$. Since $t_{i} C_{i}^{\prime}+\left(1-t_{i}\right) C_{i} \succeq C_{i}, \forall t_{i} \in[0,1]$, by choosing $t_{i}$ small, one may assume w.l.o.g. that $C_{i}^{\prime}$ is ranked in the same order as $C_{i}$. Hence, $E_{\pi_{i}}\left[U_{i}\left(C_{i}^{\prime}\right)\right]=E_{\nu_{i}}\left[U_{i}\left(C_{i}^{\prime}\right)\right]$ for all $i$, which contradicts the fact that $\left(C_{i}\right)_{i=1}^{n}$ is a P.O. of $\mathcal{E}_{2}$.

(ii) Assume there exists a feasible allocation $\left(C_{i}^{\prime}\right)_{i=1}^{n}$ such that $E_{\nu_{i}}\left[U_{i}\left(C_{i}^{\prime}\right)\right] \geq$ $E_{\nu_{i}}\left[U_{i}\left(C_{i}\right)\right]$ with a strict inequality for at least some $i$. Then, $E_{\pi_{i}}\left[U_{i}\left(C_{i}^{\prime}\right)\right] \geq$ $E_{\pi_{i}}\left[U_{i}\left(C_{i}\right)\right]$ with a strict inequality for at least some $i$, which leads to a contradiction.

We now illustrate the implications of this proposition on a simple example.

Example 3.1 Consider an economy with two agents, two states and one good, that thus can be represented in an Edgeworth box. Divide the latter into three zones:

- zone (1), where $C_{1}^{1}>C_{1}^{2}$ and $C_{2}^{1}<C_{2}^{2}$

- zone (2), where $C_{1}^{1}<C_{1}^{2}$ and $C_{2}^{1}<C_{2}^{2}$

- zone (3), where $C_{1}^{1}<C_{1}^{2}$ and $C_{2}^{1}>C_{2}^{2}$

In zone (1), everything is as if agent 1 had probability $\left(\nu_{1}^{1}, 1-\nu_{1}^{1}\right)$ and agent 2 , probability $\left(1-\nu_{2}^{2}, \nu_{2}^{2}\right)$. In zone $(2)$, agent 1 uses $\left(1-\nu_{1}^{2}, \nu_{1}^{2}\right)$ and agent $2,\left(1-\nu_{2}^{2}, \nu_{2}^{2}\right)$, while in zone $(3)$, agent 1 uses $\left(1-\nu_{1}^{2}, \nu_{1}^{2}\right)$ and agent $2,\left(\nu_{2}^{1}, 1-\nu_{2}^{1}\right)$.

In order to use $(i i)$ of proposition 3.3, we draw the three contract curves, corresponding to the P.O. in the vNM economies in which agents have the same utility index $U_{i}$ and the three possible couples of probability. Label $(a),(b)$ and $(c)$ these curves.

One notices that curve $(a)$, which is the P.O. of the vNM economy for agents having beliefs $\left(\nu_{1}^{1}, 1-\nu_{1}^{1}\right)$ and $\left(1-\nu_{2}^{2}, \nu_{2}^{2}\right)$ respectively, does not intersect zone (1), which is the zone where CEU agents do use these probability distributions as well. Hence, no points are at the same time P.O. of that vNM economy and such that $E_{\nu_{i}}\left[U_{i}\left(C_{i}\right)\right]=E_{\pi_{i}}\left[U_{i}\left(C_{i}\right)\right], i=1,2$. The same is true for curve $(c)$ and zone (3). On the other hand, part of curve $(b)$ is 
Figure 1:
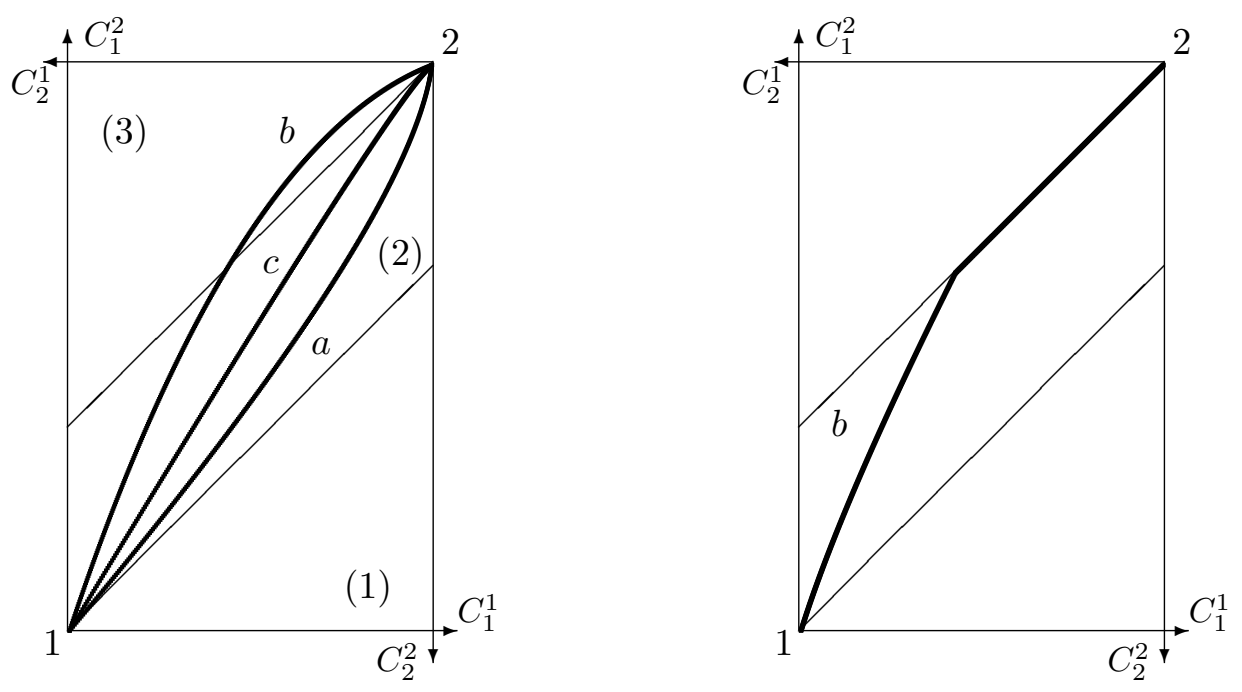

contained in zone (2). That part constitutes a subset of the set of P.O. that we are looking for. We will show later on that, in order to get the full set of P.O. of the CEU economy, one has to replace the part of curve $(b)$ that lies in zone (3) by the segment along the diagonal of agent 2 .

It follows from proposition 3.3 that, without any knowledge on the set of P.O., one has to compute the P.O. of $(k !)^{n}-1$ economies (if there are $k$ ! extremal points in $\operatorname{core}\left(\nu_{i}\right)$ for all $\left.i\right)$. Thus, the actual characterization of the set of P.O. of $\mathcal{E}_{2}$ might be somewhat tedious without further information.

In the comonotone case however, the characterization of P.O. is simpler, even though it remains partial.

Corollary 3.2 Assume $w^{1} \leq w^{2} \leq \ldots \leq w^{k}$.

(i) Let $\mathbf{U} 1$ hold and $\left(C_{i}\right)_{i=1}^{n} \in \mathbb{R}_{+}^{k n}$ be a comonotone P.O. of $\mathcal{E}_{2}$ such that $C_{i}^{1}<C_{i}^{2}<\ldots<C_{i}^{k}$ for all $i=1, \ldots, n$. Then, $\left(C_{i}\right)_{i=1}^{n}$ is a P.O. allocation of the economy in which agents are vNM maximizers with utility index $U_{i}$ and probability $\pi_{i}^{j}=\nu_{i}(\{j, \ldots, k\})-\nu_{i}(\{j+1, \ldots, k\})$ for $j<k$ and $\pi_{i}^{k}=\nu_{i}(\{k\})$.

(ii) Let $\left(C_{i}\right)_{i=1}^{n} \in \mathbb{R}_{+}^{k n}$ be a P.O. of the economy in which agents are $v N M$ maximizers with utility index $U_{i}$ and probability $\pi_{i}^{j}=\nu_{i}(\{j, \ldots, k\})-$ $\nu_{i}(\{j+1, \ldots, k\})$ for $j<k$ and $\pi_{i}^{k}=\nu_{i}(\{k\})$. If $\left(C_{i}\right)_{i=1}^{n}$ is comonotone, then it is a P.O. of $\mathcal{E}_{2}$. 
These results may now be used for equilibrium analysis as follows.

Proposition 3.4 Assume $w^{1} \leq w^{2} \leq \ldots \leq w^{k}$.

(i) Let $\left(p^{\star}, C^{\star}\right)$ be an equilibrium of $\mathcal{E}_{2}$. If $0<C_{i}^{\star 1}<\ldots<C_{i}^{\star k}$ for all $i$, then $\left(p^{\star}, C^{\star}\right)$ is an equilibrium of the economy in which agents are $v N M$ maximizers with utility index $U_{i}$ and probability $\pi_{i}^{j}=\nu_{i}(\{j, \ldots, k\})-\nu_{i}(\{j+$ $1, \ldots, k\})$ for $j<k$ and $\pi_{i}^{k}=\nu_{i}(\{k\})$.

(ii) Let $\left(p^{\star}, C^{\star}\right)$ be an equilibrium of the economy in which agents are $v N M$ maximizers with utility index $U_{i}$ and probability $\pi_{i}^{j}=\nu_{i}(\{j, \ldots, k\})-$ $\nu_{i}(\{j+1, \ldots, k\})$ for $j<k$ and $\pi_{i}^{k}=\nu_{i}(\{k\})$. If $C^{\star}$ is comonotone, then $\left(p^{\star}, C^{\star}\right)$ is an equilibrium of $\mathcal{E}_{2}$.

Proof: (i) Since $\left(p^{\star}, C^{\star}\right)$ is an equilibrium of $\mathcal{E}_{2}$, and since $v_{i}$ is differentiable at $C_{i}^{\star}$ for every $i$, there exists a multiplier $\lambda_{i}$ such that $p^{\star}=$ $\lambda_{i}\left[U_{i}^{\prime}\left(C_{i}^{\star 1}\right) \pi_{i}^{1}, \ldots, U_{i}^{\prime}\left(C_{i}^{\star k}\right) \pi_{i}^{k}\right]$, where $\pi_{i}^{j}=\nu_{i}(\{j, \ldots, k\})-\nu_{i}(\{j+1, \ldots, k\})$ for $j<k$ and $\pi_{i}^{k}=\nu_{i}(\{k\})$ for all $i$. Hence, $\left(p^{\star}, C^{\star}\right)$ is an equilibrium of the economy in which agents are vNM maximizers with probability $\left(\pi_{i}^{j}\right)$ for all $i, j$.

(ii) Let $\left(p^{\star}, C^{\star}\right)$ be an equilibrium of the economy in which agents are vNM maximizers with probability $\left(\pi_{i}^{j}\right)$ for all $i, j$. Assume $C^{\star}$ is comonotone. We thus have

$$
p^{\star} C_{i}^{\prime} \leq p^{\star} w_{i} \Rightarrow E_{\pi_{i}}\left[U_{i}\left(C_{i}^{\prime}\right)\right] \leq E_{\pi_{i}}\left[U_{i}\left(C_{i}^{\star}\right)\right]
$$

Since $E_{\nu_{i}}\left[U_{i}\left(C_{i}^{\prime}\right)\right] \leq E_{\pi_{i}}\left[U_{i}\left(C_{i}^{\prime}\right)\right]$ and $E_{\nu_{i}}\left[U_{i}\left(C_{i}^{\star}\right)\right]=E_{\pi_{i}}\left[U_{i}\left(C_{i}^{\star}\right)\right]$, we get $E_{\nu_{i}}\left[U_{i}\left(C_{i}^{\prime}\right)\right] \leq E_{\nu_{i}}\left[U_{i}\left(C_{i}^{\star}\right)\right]$ for all $i$, which implies that $\left(C^{\star}, p^{\star}\right)$ is an equilibrium of $\mathcal{E}_{2}$.

Observe that, even though the characterization of P.O. allocations is made simpler when we know that these allocations are comonotone, the above proposition does not give a complete characterization. comonotonicity of the P.O. allocations is also useful for equilibrium analysis. This leads us to look for conditions on the economy under which P.O. are comonotone.

\section{Optimal risk-sharing and equilibrium in some particular cases}

In this section, we focus on two particular cases in which we can prove directly that P.O. allocations are comonotone. 


\subsection{Convex transform of a probability distribution}

In this sub-section, we show how one can use the previous results when agents' capacities are the convex transform of a given probability distribution. In this case, one can directly apply corollary 3.2 and proposition 3.4 to get a characterization of P.O. and equilibrium.

Let $\pi=\left(\pi^{1}, \ldots, \pi^{k}\right)$ be a probability distribution on $S$, with $\pi^{j}>0$ for all $j$.

Proposition 4.1 Assume $w^{1} \leq w^{2} \leq \ldots \leq w^{k}$. Assume that, for all $i, U_{i}$ is differentiable and $\nu_{i}=f_{i} \circ \pi$, where $f_{i}$ is a strictly increasing and convex function from $[0,1]$ to $[0,1]$ with $f_{i}(0)=0, f_{i}(1)=1$. Then, at a P.O., $C_{i}^{1} \leq C_{i}^{2} \leq \ldots \leq C_{i}^{k}$ for all $i$.

Proof: Since $U_{i}$ is differentiable, strictly increasing and strictly concave, and $f_{i}$ is a strictly increasing, convex function for all $i$, it results from corollary 2 in Chew, Karni and Safra [1987] that every agent strictly respects second order stochastic dominance.

Therefore it remains to show that if every agent strictly respects second order stochastic dominance, then, at a P.O., $C_{i}^{1} \leq C_{i}^{2} \leq \ldots \leq C_{i}^{k}$ for all $i$. We do so using proposition 4.1 in Chateauneuf, Cohen and Kast [1997].

Assume $\left(C_{i}\right)_{i=1}^{n}$ is not comonotone. W.l.o.g., assume that $C_{1}^{1}>C_{1}^{2}$, $C_{2}^{1}<C_{2}^{2}$, and $C_{1}^{1}+C_{2}^{1} \leq C_{1}^{2}+C_{2}^{2}$. Let $C^{\prime}$ be such that:

$$
C_{1}^{1 \prime}=C_{1}^{2 \prime}=\frac{\pi^{1} C_{1}^{1}+\pi^{2} C_{1}^{2}}{\pi^{1}+\pi^{2}} \quad \text { and } \quad C_{1}^{j \prime}=C_{1}^{j}, \quad j>2
$$

Let $C_{i}^{\prime}=C_{i}$ for all $i>2$, and $C_{2}^{\prime}$ be determined by the feasibility condition $C_{1}+C_{2}=C_{1}^{\prime}+C_{2}^{\prime}$. Hence,

$C_{2}^{1 \prime}=C_{2}^{1}+\frac{\pi^{2}}{\pi^{1}+\pi^{2}}\left(C_{1}^{1}-C_{1}^{2}\right), \quad C_{2}^{2 \prime}=C_{2}^{2}-\frac{\pi^{1}}{\pi^{1}+\pi^{2}}\left(C_{1}^{1}-C_{1}^{2}\right)$ and $C_{2}^{j \prime}=C_{2}^{j}, j>2$

It may easily be checked that $C_{1}^{2}<C_{1}^{1 \prime}=C_{1}^{2 \prime}<C_{1}^{1}$, and $C_{2}^{1}<C_{2}^{1 \prime} \leq C_{2}^{2 \prime}<$ $C_{2}^{2}$. Furthermore, $\pi^{1} C_{1}^{1 \prime}+\pi^{2} C_{1}^{2 \prime}=\pi^{1} C_{1}^{1}+\pi^{2} C_{1}^{2}$, and $\pi^{1} C_{2}^{1 \prime}+\pi^{2} C_{2}^{2 \prime}=$ $\pi^{1} C_{2}^{1}+\pi^{2} C_{2}^{2}$.

Therefore, $C_{i}^{\prime} i=1,2$ is a strictly less risky allocation than $C_{i} i=1,2$, with respect to mean preserving increases in risk. It follows that agents 1 and 2 are strictly better off with $C^{\prime}$, while other agents' utilities are unaffected. Hence, $C^{\prime}$ Pareto dominates $C$. Thus, any P.O. $C$ must be comonotone, i.e., $C_{i}^{1} \leq C_{i}^{2} \leq \ldots \leq C_{i}^{k}$ for all $i$. 
Using corollary 3.2, we can then provide a partial characterization of the set of P.O. Note that such a characterization was not provided by the analysis in Chateauneuf, Cohen and Kast [1997] or Landsberger and Meilijson [1994].

Proposition 4.2 Assume $w^{1} \leq \ldots \leq w^{k}$ and that agents are CEU maximizers with $\nu_{i}=f_{i} \circ \pi, f_{i}$ convex, strictly increasing and such that $f_{i}(0)=0$ and $f_{i}(1)=1$. Then,

(i) Let $\left(C_{i}\right)_{i=1}^{n} \in \mathbb{R}_{+}^{k n}$ be a P.O. of this economy such that $C_{i}^{1}<C_{i}^{2}<$ $\ldots<C_{i}^{k}$ for all $i=1, \ldots, n$. Then, $\left(C_{i}\right)_{i=1}^{n}$ is a P.O. allocation of the economy in which agents are vNM maximizers with utility index $U_{i}$ and probability $\pi_{i}^{j}=\left[f_{i}\left(\sum_{s=j}^{k} \pi^{s}\right)-f_{i}\left(\sum_{s=j+1}^{k} \pi^{s}\right)\right]$ for $j=1, \ldots, k-1$, and $\pi_{i}^{k}=f_{i}\left(\pi^{k}\right)$.

(ii) Let $\left(C_{i}\right)_{i=1}^{n} \in \mathbb{R}_{+}^{k n}$ be a P.O. of the economy in which agents are $v N M$ maximizers with utility index $U_{i}$ and probability $\pi_{i}^{j}=\left[f_{i}\left(\sum_{s=j}^{k} \pi^{s}\right)-f_{i}\left(\sum_{s=j+1}^{k} \pi^{s}\right)\right]$ for $j=1, \ldots, k-1$, and $\pi_{i}^{k}=f_{i}\left(\pi^{k}\right)$. If $\left(C_{i}\right)_{i=1}^{n}$ is comonotone, then it is a P.O. of the CEU economy with $\nu_{i}=f_{i} \circ \pi$.

Proof: See corollary 3.2.

The same type of result can be deduced for equilibrium analysis from proposition 3.4, and we omit its formal statement here.

The previous characterization formally includes the Rank-DependentExpected-Utility model introduced by Quiggin [1982] in the case of (probabilized) risk. It also applies to so-called "simple capacities" (see e.g. Dow and Werlang [1992]), which are particularly easy to deal with in applications.

Indeed, let agents have the following simple capacities: $\nu_{i}(A)=(1-$ $\left.\xi_{i}\right) \pi(A)$ for all $A \in \mathcal{A}, A \neq S$, and $\nu_{i}(S)=1$, where $\pi$ is a given probability measure with $0<\pi^{j}<1$ for all $j$, and $0 \leq \xi<1$.

These capacities can be written $\nu_{i}=f_{i} \circ \pi$ where $f_{i}$ is such that $f_{i}(0)=0$, $f_{i}(1)=1$, is strictly increasing, continuous and convex, with:

$$
\left\{\begin{array}{l}
f_{i}(p)=\left(1-\xi_{i}\right) p \quad \text { if } 0 \leq p \leq \max _{\{\pi(A)<1\}} \pi(A) \\
f_{i}(1)=1
\end{array}\right.
$$

Hence, $\nu_{i}$ is a convex transformation of $\pi$, and we can apply the results of this sub-section to characterize the set of P.O. in an economy where all agents have such simple capacities. 


\subsection{The two-state case}

We restrict our attention here to the case $S=\{1,2\}$. Agent $i$ has a capacity $\nu_{i}$ characterized by two numbers $\nu_{i}(\{1\}), \nu_{i}(\{2\})$ such that $\nu_{i}(\{1\}) \leq 1-$ $\nu_{i}(\{2\})$. To simplify notation, we'll denote $\nu_{i}(\{s\})=\nu_{i}^{s}$. In this particular case, $\operatorname{core}\left(\nu_{i}\right)=\left\{(\pi, 1-\pi) \mid \pi \in\left[\nu_{i}^{1}, 1-\nu_{i}^{2}\right]\right\}$.

Call $\mathcal{E}_{3}$ the two-state exchange economy in which agents are CEU maximizers with capacity $\nu_{i}$ and utility index $U_{i}, i=1, \ldots, n$.

Assumption C: $\cap_{i} \operatorname{core}\left(\nu_{i}\right) \neq \emptyset$

This assumption is equivalent to $\nu_{i}^{1}+\nu_{j}^{2} \leq 1, i, j=1, \ldots, n$, or stated differently, to $\cap_{i}\left[\nu_{i}^{1}, 1-\nu_{i}^{2}\right] \neq \emptyset$. Recall that in the two-state case, under $\mathbf{C}$, agents' capacities are convex.

We now proceed to show that this "minimal consensus" assumption is enough to show that P.O. are comonotone.

Proposition 4.3 Let $\mathbf{C}$ hold. Then, P.O. are comonotone.

Proof: Assume $w^{1} \leq w^{2}$ and $C$ not comonotone. W.l.o.g., assume that $C_{1}^{1}>C_{1}^{2}, C_{2}^{1}<C_{2}^{2}$. Let $(\pi, 1-\pi) \in \cap_{i} \operatorname{core}\left(\nu_{i}\right)$ and $C^{\prime}$ be the feasible allocation defined by

$$
C_{1}^{1 \prime}=C_{1}^{2 \prime}=\pi C_{1}^{1}+(1-\pi) C_{1}^{2}
$$

and $C_{2}^{1 \prime}$ and $C_{2}^{2 \prime}$ are such that $C_{1}^{j \prime}+C_{2}^{j \prime}=C_{1}^{j}+C_{2}^{j}, j=1,2$, i.e.

$$
C_{2}^{1 \prime}=C_{2}^{1}+(1-\pi)\left(C_{1}^{1}-C_{1}^{2}\right), \quad C_{2}^{2 \prime}=C_{2}^{2}-\pi\left(C_{1}^{1}-C_{1}^{2}\right)
$$

One obviously has $C_{2}^{1}<C_{2}^{1 \prime} \leq C_{2}^{2 \prime}<C_{2}^{2}$.

Finally, let $C_{i}^{j \prime}=C_{i}^{j}, \quad \forall i>2, j=1,2$. We now prove that $C^{\prime}$ Pareto dominates $C$.

$$
\begin{aligned}
v_{1}\left(C_{1}^{\prime}\right)-v_{1}\left(C_{1}\right) & =U_{1}\left(\pi C_{1}^{1}+(1-\pi) C_{1}^{2}\right)-\nu_{1}^{1} U_{1}\left(C_{1}^{1}\right)-\left(1-\nu_{1}^{1}\right) U_{1}\left(C_{1}^{2}\right) \\
& >\left(\pi-\nu_{1}^{1}\right)\left(U_{1}\left(C_{1}^{1}\right)-U_{1}\left(C_{1}^{2}\right)\right) \geq 0
\end{aligned}
$$

since $U_{1}$ is strictly concave and $\pi \geq \nu_{i}^{1}$. Now, consider agent 2's utility:

$$
v_{2}\left(C_{2}^{\prime}\right)-v_{2}\left(C_{2}\right)=\left(1-\nu_{2}^{2}\right)\left[U_{2}\left(C_{2}^{1 \prime}\right)-U_{2}\left(C_{2}^{1}\right)\right]+\nu_{2}^{2}\left[U_{2}\left(C_{2}^{2 \prime}\right)-U_{2}\left(C_{2}^{2}\right)\right]
$$


Since $U_{2}$ is strictly concave and $C_{2}^{1}<C_{2}^{1 \prime} \leq C_{2}^{2 \prime}<C_{2}^{2}$, we have:

$$
\frac{U_{2}\left(C_{2}^{1 \prime}\right)-U_{2}\left(C_{2}^{1}\right)}{C_{2}^{1 \prime}-C_{2}^{1}}>\frac{U_{2}\left(C_{2}^{2}\right)-U_{2}\left(C_{2}^{2 \prime}\right)}{C_{2}^{2}-C_{2}^{2 \prime}}
$$

and hence, $\frac{U_{2}\left(C_{2}^{1 \prime}\right)-U_{2}\left(C_{2}^{1}\right)}{1-\pi}>\frac{U_{2}\left(C_{2}^{2}\right)-U_{2}\left(C_{2}^{2 \prime}\right)}{\pi}$. Therefore,

$$
v_{2}\left(C_{2}^{\prime}\right)-v_{2}\left(C_{2}\right)>\left[\left(1-\nu_{2}^{2}\right) \frac{1-\pi}{\pi}-\nu_{2}^{2}\right]\left[U_{2}\left(C_{2}^{2}\right)-U_{2}\left(C_{2}^{2 \prime}\right)\right] \geq 0
$$

since $\left(1-\nu_{2}^{2}\right)(1-\pi)-\pi \nu_{2}^{2}=1-\nu_{2}^{2}-\pi \geq 0$ and $U_{2}\left(C_{2}^{2}\right)-U_{2}\left(C_{2}^{2 \prime}\right)>0$. Hence, $C^{\prime}$ Pareto dominates $C$.

Remark: If $\nu_{i}^{1}+\nu_{j}^{2}<1, i, j=1, \ldots, n$, which is equivalent to the assumption that $\cap_{i} \operatorname{core}\left(\nu_{i}\right)$ contains more than one element, then one can extend proposition 4.3 to linear utilities.

Remark: Although convex capacities can, in the two-state case, be expressed as simple capacities, the analysis of sub-section 4.1 (and in particular proposition 4.1), cannot be used here. Indeed, assumption $\mathbf{C}$ does not require that agents' capacities are all a convex transform of the same probability distribution as example 4.1 shows.

Example 4.1 There are two agents with capacity $\nu_{1}^{1}=1 / 3, \nu_{1}^{2}=2 / 3$, and $\nu_{2}^{1}=1 / 6, \nu_{2}^{2}=2 / 3$ respectively. Assumption $\mathbf{C}$ is satisfied since $\pi=$ $(1 / 3,2 / 3)$ is in the intersection of the cores. The only way $\nu_{1}$ and $\nu_{2}$ could be a convex transform of the same probability distribution is $\nu_{1}=\pi$ and $\nu_{2}=f_{2} \circ \pi$ with $f_{2}(1 / 3)=1 / 6$ and $f_{2}(2 / 3)=2 / 3$. But $f_{2}$ then fails to be convex.

Intuition derived from proposition 4.3 might suggest that some minimal consensus assumption might be enough to prove comonotonicity of the P.O. However, that intuition is not valid in general, as can be seen in the following example, in which the intersection of the cores of the capacities is non-empty, but where (some) P.O. allocations are not comonotone.

Example 4.2 There are two agents, with the same utility index $U_{i}(C)=$ $2 C^{1 / 2}$, but different beliefs. The latter are represented by two convex capacities defined as follows:

$$
\begin{array}{ccc}
\nu_{1}(\{1\})=\frac{3}{9} & \nu_{1}(\{2\})=\frac{3}{9} & \nu_{1}(\{3\})=\frac{1}{9} \\
\nu_{1}(\{1,2\})=\frac{6}{9} & \nu_{1}(\{1,3\})=\frac{6}{9} & \nu_{1}(\{2,3\})=\frac{4}{9}
\end{array}
$$




$$
\begin{array}{ccc}
\nu_{2}(\{1\})=\frac{2}{9} & \nu_{2}(\{2\})=\frac{2}{9} & \nu_{2}(\{3\})=\frac{3}{9} \\
\nu_{2}(\{1,2\})=\frac{4}{9} & \nu_{2}(\{1,3\})=\frac{5}{9} & \nu_{2}(\{2,3\})=\frac{5}{9}
\end{array}
$$

The intersection of the cores of these two capacities is non-empty since the probability defined by $\pi^{j}=1 / 3, j=1,2,3$ belongs to both cores. The endowment in each state is respectively $w^{1}=1, w^{2}=12$, and $w^{3}=13$. We consider the optimal allocation associated to the weights $(1 / 2,1 / 2)$ and show it cannot be comonotone. In order to do that, we show that the maximum of $v_{1}\left(C_{1}\right)+v_{2}\left(C_{2}\right)$ subject to the constraints $C_{1}^{j}+C_{2}^{j}=w^{j}, j=1,2,3$ and $C_{i}^{j} \geq 0$ for all $i$ and $j$, does not obtain for $C_{i}^{1} \leq C_{i}^{2} \leq C_{i}^{3}, i=1,2$.

Observe first that if $C_{i}^{1} \leq C_{i}^{2} \leq C_{i}^{3}, i=1,2$, then:

$v_{1}\left(C_{1}\right)+v_{2}\left(C_{2}\right)=2\left(\frac{5}{9} \sqrt{C_{1}^{1}}+\frac{3}{9} \sqrt{C_{1}^{2}}+\frac{1}{9} \sqrt{C_{1}^{3}}+\frac{4}{9} \sqrt{C_{2}^{1}}+\frac{2}{9} \sqrt{C_{2}^{2}}+\frac{3}{9} \sqrt{C_{2}^{3}}\right)$

Call $g\left(C_{1}^{1}, C_{1}^{2}, C_{1}^{3}, C_{2}^{1}, C_{2}^{2}, C_{2}^{3}\right)$ the above expression. Note that $v_{1}\left(C_{1}\right)+$ $v_{2}\left(C_{2}\right)$ takes the exact same form if $C_{1}^{1}<C_{1}^{3}<C_{1}^{2}$ and $C_{2}^{1}<C_{2}^{2}<C_{2}^{3}$.

The optimal solution to the maximization problem:

$$
\begin{aligned}
\max & g\left(C_{1}^{1}, C_{1}^{2}, C_{1}^{3}, C_{2}^{1}, C_{2}^{2}, C_{2}^{3}\right) \\
\text { s.t. } & \left\{\begin{array}{rl}
C_{1}^{j}+C_{2}^{j}=w^{j} & j=1,2,3 \\
C_{i}^{j} \geq 0 & j=1,2,3 \quad i=1,2
\end{array}\right.
\end{aligned}
$$

is $\left(\widehat{C}_{1}^{1}, \widehat{C}_{1}^{2}, \widehat{C}_{1}^{3}\right)=\left(\frac{25}{41}, \frac{108}{13}, \frac{13}{10}\right)$ and $\left(\widehat{C}_{2}^{1}, \widehat{C}_{2}^{2}, \widehat{C}_{2}^{3}\right)=\left(\frac{16}{41}, \frac{48}{13}, \frac{117}{10}\right)$. It satisfies $0<\widehat{C}_{1}^{1}<\widehat{C}_{1}^{3}<\widehat{C}_{1}^{2}$ and $0<\widehat{C}_{2}^{1}<\widehat{C}_{2}^{2}<\widehat{C}_{2}^{3}$. Therefore:

$v_{1}\left(\widehat{C}_{1}\right)+v_{2}\left(\widehat{C}_{2}\right)>v_{1}\left(C_{1}\right)+v_{2}\left(C_{2}\right)$ for all $C$ such that $C_{i}^{1} \leq C_{i}^{2} \leq C_{i}^{3}, i=1,2$

and hence the P.O. associated to equal weights for each agent is not comonotone. $\diamond$

One may expect that it follows from proposition 4.3 that P.O. of $\mathcal{E}_{3}$ are the P.O. of the $\mathrm{vNM}$ economy in which agents have probability $\pi_{i}=$ $1-\nu_{i}^{2}, i=1, \ldots, n$. However, it is not so, since as recalled in sub-section 2.4, P.O. of a vNM economy with different beliefs are not in general comonotone. We can nevertheless use proposition 3.3 to provide a partial characterization of the set of P.O.

In this particular case of only two states, we can obtain a full characterization of the set of P.O. if there are only two agents in the economy. This should then be interpreted as a characterization of the optimal risk-sharing arrangement among two parties to a contract (arrangement that has been widely studied in the vNM case). 
Proposition 4.4 Assume $n=2, w^{1}<w^{2}$ and that agents have capacities $\nu_{i}, i=1,2$ which fulfill $\mathbf{C}$, and such that $\nu_{1}^{2}<\nu_{2}^{2}$. Assume finally $\mathbf{U 1}$ and let $\left(C_{i}\right)_{i=1,2}$ be a P.O. of $\mathcal{E}_{3}$. Then, there are only two cases:

(i) Either $C_{i}^{1}<C_{i}^{2}, \forall i$ and $\left(C_{i}\right)_{i=1,2}$ is a P.O. of the vNM economy with utility index $U_{i}$ and probabilities $\pi_{i}=1-\nu_{i}^{2}, i=1,2$.

(ii) Or, $C_{1}^{1}=C_{1}^{2}, C_{2}^{1}<C_{2}^{2}$ and

$$
\frac{\nu_{1}^{2}}{1-\nu_{1}^{2}} \leq \frac{U_{2}^{\prime}\left(C_{2}^{2}\right)}{U_{2}^{\prime}\left(C_{2}^{1}\right)} \frac{\nu_{2}^{2}}{1-\nu_{2}^{2}}
$$

Proof: It follows from proposition 4.3 that there are three cases : $C_{i}^{1}<$ $C_{i}^{2}, \forall i, C_{1}^{1}=C_{1}^{2}$ and $C_{2}^{1}<C_{2}^{2}$ and lastly $C_{2}^{1}=C_{2}^{2}$ and $C_{1}^{1}<C_{1}^{2}$.

The first case follows from corollary 3.2 .

Second, if $0<C_{1}^{1}=C_{1}^{2},\left(C_{i}\right)_{i=1,2}$ is optimal iff there exists $t>0$ such that $\nabla v_{2}\left(C_{2}\right) \in t \partial v_{1}\left(C_{1}\right)$ which is equivalent to

$$
\frac{\nu_{1}^{2}}{1-\nu_{1}^{2}} \leq \frac{U_{2}^{\prime}\left(C_{2}^{2}\right)}{U_{2}^{\prime}\left(C_{2}^{1}\right)} \frac{\nu_{2}^{2}}{1-\nu_{2}^{2}} \leq \frac{1-\nu_{1}^{1}}{\nu_{1}^{1}}
$$

Since $\mathbf{C}$ is fulfilled, $1 \leq \frac{\left(1-\nu_{1}^{1}\right)\left(1-\nu_{2}^{2}\right)}{\nu_{1}^{1} \nu_{2}^{2}}$; hence the right-hand side of (2) is fulfilled and (1) is equivalent to (2).

Lastly, the case $0<C_{2}^{1}=C_{2}^{2}$ is symmetric. The first-order corresponding conditions imply $\nu_{1}^{2} \geq \nu_{2}^{2}$ which contradicts our hypothesis.

We can illustrate the optimal risk-sharing arrangement just derived in an Edgeworth box. Figure $2(a)$ represents case $(i)$ and the optimal contract is the same as the one in the associated vNM setup.

However, figure $2(b)$ gives a different risk-sharing rule, that can interpreted as follows. The assumption $\nu_{1}^{2}<\nu_{2}^{2}$ is equivalent to $E_{\nu_{1}}(x) \leq E_{\nu_{2}}(x)$ for all $x$ comonotone with $w$. But we have just shown that we could restrict our attention to allocations that are comonotone with $w$. Hence, the assumption $\nu_{1}^{2}<\nu_{2}^{2}$ can be interpreted as a form of pessimism of agent 1 . Under that assumption, agent 2 never insures himself completely, whereas agent 1 might insure do so. This is incompatible with a vNM economy with strictly concave and differentiable utility indices.

Finally, risk-sharing arrangements such as the one represented on figure 3 (a) cannot be excluded a priori, i.e., there is no reason that the contract curve in the vNM economy crosses agent 1's diagonal only once. On figure 
Figure 2:

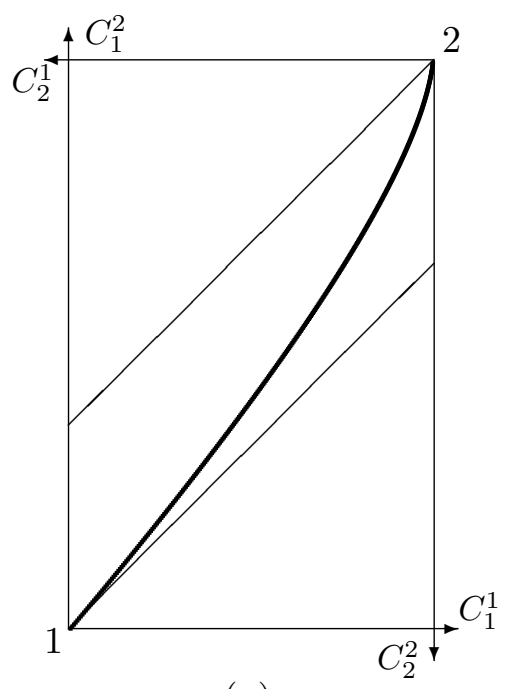

(a)

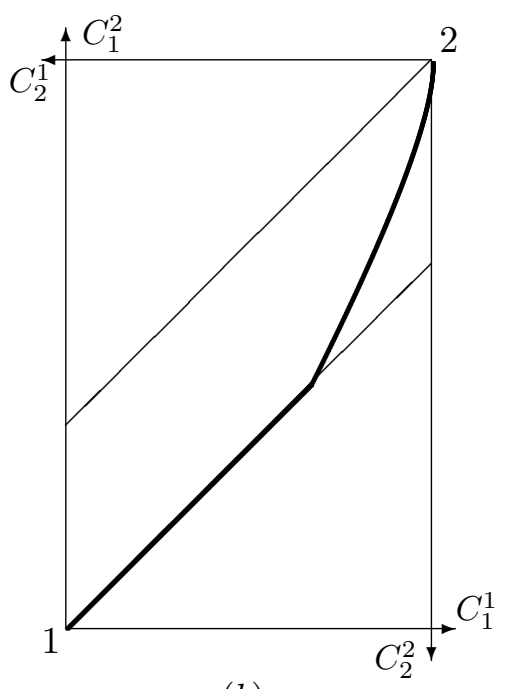

(b)

$3(a)$, the thin line represents P.O. of the vNM economy in which agent $i$ uses probability $\left(1-\nu_{i}^{2}, \nu_{i}^{2}\right)$ that are not P.O. of the CEU economy, as they are not comonotone.

If agent 2 has a utility index of the DARA type, it is easy to show that the set of P.O. of the economy in which agents have utility index $U_{i}$ and probability $\left(1-\nu_{i}^{2}, \nu_{i}^{2}\right)$ crosses agent 1 's diagonal at most once, hence preventing the kind of situation represented on figure 3.

When there are only two (types of) agents, we can also go further in the characterization of the set of equilibria.

Proposition 4.5 Assume $k=2, n=2, w^{1} \leq w^{2}, \mathbf{C}$ and $\mathbf{U 1}$ hold and $\nu_{1}^{2}<\nu_{2}^{2}$. Let $\left(p^{\star}, C^{\star}\right)$ be an equilibrium of $\mathcal{E}$. Then there are only two cases:

(i) Either $C_{i}^{1 \star}<C_{i}^{2 \star}, i=1,2$ and $\left(p^{\star}, C^{\star}\right)$ is an equilibrium of a vNM economy in which agents have utility index $U_{i}$ and beliefs given by $\pi_{i}=$ $1-\nu_{i}^{2}, i=1,2$.

(ii) Or, $C_{1}^{1 \star}=C_{1}^{2 \star}=C^{\star \star}$ and $C^{\star \star}$ satisfies the following :

(a) $\quad\left(1-\nu_{2}^{2}\right)\left(w_{1}^{1}-C^{\star \star}\right) U_{2}^{\prime}\left(w^{1}-C^{\star \star}\right)+\nu_{2}^{2}\left(w_{1}^{2}-C^{\star \star}\right) U_{2}^{\prime}\left(w^{2}-C^{\star \star}\right)=0$

(b) $\quad \frac{\nu_{1}^{2}}{1-\nu_{1}^{2}} \leq \frac{-\left(w_{1}^{1}-C^{\star \star}\right)}{\left(w_{1}^{2}-C^{\star \star}\right)}$

Proof: It follows from proposition 4.4 that either $C_{i}^{1 \star}<C_{i}^{2 \star}, i=1,2$ or $C_{1}^{1 \star}=C_{1}^{2 \star}=C^{\star \star}$. The first case follows from proposition 3.4. In the 
Figure 3:

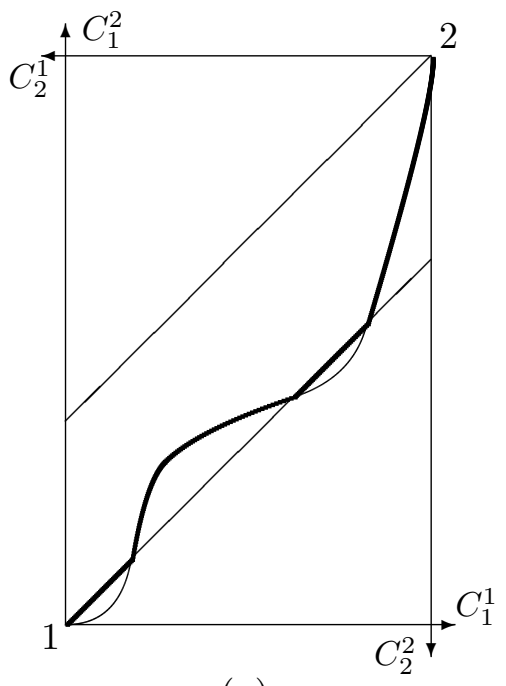

(a)

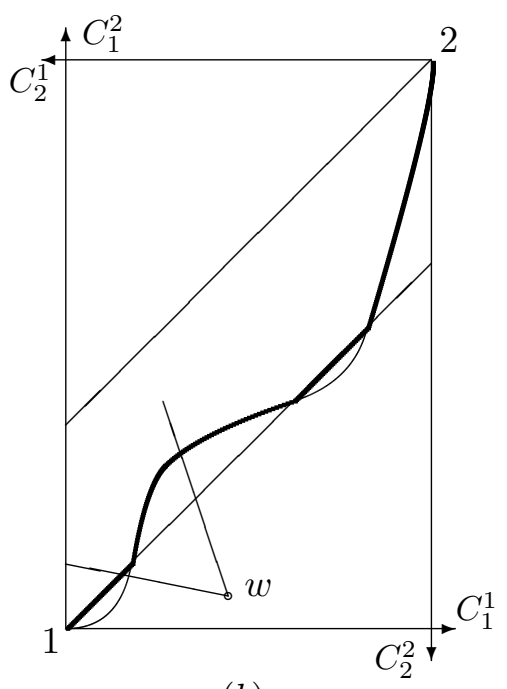

(b)

second case, the P.O. allocation is supported by the price $\left(\left(1-\nu_{2}^{2}\right) U_{2}^{\prime}\left(w^{1}-\right.\right.$ $\left.\left.C^{\star \star}\right), \nu_{2}^{2} U_{2}^{\prime}\left(w^{2}-C^{\star \star}\right)\right)$, hence the tangent to agent two's indifference curve at $\left(w^{1}-C^{\star \star}, w^{2}-C^{\star \star}\right)$ has the following equation in the $\left(C^{1}, C^{2}\right)$ plane:

$$
\left(1-\nu_{2}^{2}\right) U_{2}^{\prime}\left(w^{1}-C^{\star \star}\right)\left(C^{1}-C^{\star \star}\right)+\nu_{2}^{2} U_{2}^{\prime}\left(w^{2}-C^{\star \star}\right)\left(C^{2}-C^{\star \star}\right)=0
$$

Now $C^{\star}$ is an equilibrium allocation iff $\left(w_{1}^{1}, w_{1}^{2}\right)$ fulfills that equation. Condition (b) follows from condition $(a)$ and condition $(i i)$ from proposition 4.4 .

There might therefore exist, for a range of initial endowments, equilibria at which agent 1 is perfectly insured even though agent 2 has strictly convex preferences. Observe also that nothing excludes a priori the possibility of having different kind of equilibria for the same initial endowment (see figure $3(b))$.

\section{Optimal risk-sharing and equilibrium without aggregate risk}

We now turn to the study of economies without aggregate uncertainty ${ }^{5}$. This corresponds to the case of individual risk first analyzed by Malinvaud [1972]

\footnotetext{
${ }^{5}$ For a study of optimal risk-sharing without aggregate uncertainty and an infinite state space, see Billot, Chateauneuf, Gilboa and Tallon [1998].
} 
and [1973]. A particular case is the one of a sunspot economy, in which uncertainty is purely extrinsic and does not affect the fundamentals, i.e., each agent's endowment is independent of the state of the world (see Tallon [1998] for a study of sunspot economies with CEU agents). Our analysis of the case of purely individual risk might also yield further insights as to which type of financial contracting (e.g. mutual insurance rather than trade on Arrow securities defined on individual states) is necessary in such economies to decentralize an optimal allocation.

It turns out that the economy under consideration possesses remarkable properties: P.O. are comonotone and coincide with full insurance allocations, under the relatively weak condition $\mathbf{C}$. Furthermore, this condition, which is weaker than convexity of preferences, is enough to prove existence of an equilibrium. Finally, the case of purely individual risk lends itself to the introduction of several goods.

We thus move to an economy with $m$ goods, indexed by subscript $\ell$. $C_{i \ell}^{j}$ is the consumption of good $\ell$ by agent $i$ in state $j$. We have, $C_{i}^{j}=$ $\left(C_{i 1}^{j}, \ldots, C_{i m}^{j}\right)$, and $C_{i}=\left(C_{i}^{1}, \ldots, C_{i}^{k}\right)$. If $C_{i}^{j}=C_{i}^{j^{\prime}}$ for all $j, j^{\prime}$, then $C_{i}$ will denote both this constant bundle (i.e. $C_{i}^{j} \equiv C_{i}$ ) and the vector composed of $k$ such vectors, the context making it clear which meaning is intended. Let $p_{\ell}^{j}$ be the price of good $\ell$ available in state $j, p^{j}=\left(p_{1}^{j}, \ldots, p_{m}^{j}\right)$, and $p=\left(p^{1}, \ldots, p^{k}\right)$.

The utility index $U_{i}$ is now defined on $\mathbb{R}_{+}^{m}$, and is still assumed to be strictly concave and strictly increasing. We will also need a generalization of assumption $\mathbf{U} \mathbf{1}$ to the multi-good case, that ensures that the solution to the agent's maximization program is interior.

Assumption Um: $\forall i,\left\{x^{\prime} \in \mathbb{R}_{+}^{m} \mid U_{i}\left(x^{\prime}\right) \geq U_{i}(x)\right\} \subset \mathbb{R}_{++}^{m}, \forall x \in \mathbb{R}_{++}^{m}$.

Aggregate endowment is the same across states, although its distribution among households might differ in each state. Therefore, we consider a pure exchange economy $\mathcal{E}_{4}$ with $n$ agents and $m$ goods described by the list: $\mathcal{E}_{4}=\left(v_{i}: \mathbb{R}_{+}^{k m} \rightarrow \mathbb{R}, w_{i} \in \mathbb{R}_{+}^{k m}, i=1, \ldots, n\right)$. We will denote aggregate endowments $w$, i.e., $w=\sum_{i} w_{i}$.

Before dealing with non-additive beliefs, we first recall some known results in the vNM case.

Proposition 5.1 Assume all agents have identical $v N M$ beliefs, $\pi=\left(\pi^{1}, \ldots, \pi^{k}\right)$. 
Then,

(i) At a Pareto optimum, $C_{i}^{j}=C_{i}^{j^{\prime}}$ for all $i, j, j^{\prime}$ and $\left(C_{i}^{1}\right)_{i=1}^{n}$ is a P.O. of the static economy $\left(U_{i}, \bar{w}_{i}=E_{\pi}\left(w_{i}\right), i=1, \ldots, n\right)$.

(ii) Let $\mathbf{U m}$ hold. $\left(p^{\star}, C^{\star}\right)$ is an equilibrium of the vNM economy if and only if there exists $q^{\star} \in \mathbb{R}_{+}^{m}$ such that $p^{\star}=\left(q^{\star} \pi^{1}, q^{\star} \pi^{2}, \ldots, q^{\star} \pi^{k}\right)$ and $\left(q^{\star}, C^{\star}\right)$ is an equilibrium of the static economy $\left(U_{i}, \bar{w}_{i}=E_{\pi}\left(w_{i}\right), i=1, \ldots, n\right)$.

It is also easy to see that if agents' beliefs are different, agents will consume state-dependent bundles at an optimum. We now examine to what extent these results, obtained in the vNM case, generalize to the CEU setup, assuming that condition $\mathbf{C}$ holds.

Using proposition 2.1 this assumption is equivalent ${ }^{6}$ to $\mathcal{P} \neq \emptyset$, where $\mathcal{P}=\left\{\pi \in \mathbb{R}_{++}^{k} \mid \sum_{j=1}^{k} \pi^{j}=1\right.$ and $\left.v_{i}\left(C_{i}^{1}, \ldots, C_{i}^{k}\right) \leq E_{\pi}\left(U_{i}\left(C_{i}\right)\right), \forall i, \forall C_{i}\right\}$

Recall that assumption $\mathbf{C}$ was not enough to prove comonotonicity of P.O. in the general case (though it was sufficient in the two-state case).

We now proceed to fully characterize the set of P.O.

Proposition 5.2 Let $\mathbf{C}$ hold. Then,

(i) Any P.O. $\left(C_{i}\right)_{i=1}^{n}$ of $\mathcal{E}_{4}$ is such that $C_{i}^{j}$ is independent of $j$ for all $i$ and $\left(C_{i}^{1}\right)_{i=1}^{n}$ is a P.O. of the static economy in which agents have utility function $\left(U_{i}\right)_{i=1}^{n}$.

(ii) The allocation $\left(C_{i}\right)_{i=1}^{n} \in \mathbb{R}_{+}^{k m n}$ is a P.O. of $\mathcal{E}_{4}$ if and only if it is a P.O. of a vNM economy with utility index $U_{i}$ and identical probability over the set of states of the world. Hence, P.O. are independent of the capacities.

Proof: $(i)$ Assume, to the contrary, that there exist an agent (say agent 1) and states $j$ and $j^{\prime}$ such that $C_{1}^{j} \neq C_{1}^{j^{\prime}}$. Let $\bar{C}_{i}^{j} \equiv \bar{C}_{i}=E_{\pi}\left(C_{i}\right)$ for all $j$ and all $i$ where $\pi \in \mathcal{P}$. This allocation is feasible: $\sum_{i} \bar{C}_{i}=E_{\pi}\left(\sum_{i} C_{i}\right)=w$. By definition of $\mathcal{P}, v_{i}\left(C_{i}^{1}, \ldots, C_{i}^{k}\right) \leq E_{\pi}\left(U_{i}\left(C_{i}\right)\right)$ for all $i$. Now, $E_{\pi}\left(U_{i}\left(C_{i}\right)\right) \leq$ $U_{i}\left(E_{\pi}\left(C_{i}\right)\right)=U_{i}\left(\bar{C}_{i}\right)=v_{i}\left(\bar{C}_{i}^{1}, \ldots, \bar{C}_{i}^{k}\right)$ for all $i$, since $U_{i}$ is concave. This last inequality is strict for agent 1 , since $C_{1}^{j} \neq C_{1}^{j^{\prime}}, \pi \gg 0$, and $U_{1}$ is strictly concave. Therefore, $v_{i}\left(C_{i}^{1}, \ldots, C_{i}^{k}\right) \leq v_{i}\left(\bar{C}_{1}^{1}, \ldots, \bar{C}_{i}^{k}\right)$ for all $i$, with a strict inequality for agent one, a contradiction to the fact that $\left(C_{i}\right)_{i=1}^{n}$ is an optimum of $\mathcal{E}_{4}$.

\footnotetext{
${ }^{6}$ Recall that we are dealing with capacities such that $1>\nu(A)>0$ for all $A \neq \emptyset$ and $A \neq S$.
} 
(ii) We skip the proof for this part of the proposition for it relies on the same type of argument as that of proposition 3.3.

Thus, even with different "beliefs" (in the sense of different capacities), agents might still find it optimal to fully insure themselves: differences in beliefs do not necessarily lead agents to optimally bear some risk as in the vNM case.

We now proceed to study the equilibrium set.

\section{Proposition 5.3 Let $\mathbf{C}$ hold.}

(i) Let $\left(p^{\star}, C^{\star}\right)$ be an equilibrium of a vNM economy in which all agents have utility index $U_{i}$ and beliefs given by $\pi \in \mathcal{P}$, then $\left(p^{\star}, C^{\star}\right)$ is an equilibrium of $\mathcal{E}_{4}$.

(ii) Conversely, assume $\nu_{i}$ is convex and $U_{i}$ satisfies $\mathbf{U m}$ for all $i$. Let $\left(p^{\star}, C^{\star}\right)$ be an equilibrium of $\mathcal{E}_{4}$, then there exists $\pi \in \mathcal{P}$ such that $\left(p^{\star}, C^{\star}\right)$ is an equilibrium of the $v N M$ economy in which all agents have utility index $U_{i}$ and probability $\pi$. Furthermore, $p^{\star}=\left(q^{\star} \pi^{1}, q^{\star} \pi^{2}, \ldots, q^{\star} \pi^{k}\right)$ with $\pi \in \mathcal{P}$ and $q^{\star}=\lambda_{i} \nabla U_{i}\left(C_{i}^{\star}\right), \lambda_{i} \in \mathbb{R}_{+}$for all $i$.

Proof: $(i)$ Let $\left(p^{\star}, C^{\star}\right)$ be an equilibrium of a vNM economy in which all agents have beliefs given by $\pi$. We have, $C_{i}^{j \star}=C_{i}^{j^{\prime} \star}$ for all $j, j^{\prime}$ and all $i$. By definition of an equilibrium, $\sum_{i} C_{i}^{j \star}=\sum_{i} w_{i}^{j}$ for all $j$ and, for all $i$ :

$$
C_{i}^{\prime} \geq 0, \quad p^{\star} C_{i}^{\prime} \leq p^{\star} w_{i} \Rightarrow E_{\pi}\left(U_{i}\left(C_{i}^{\prime}\right)\right) \leq E_{\pi}\left(U_{i}\left(C_{i}^{\star}\right)\right)
$$

Now, since $\pi \in \mathcal{P}, v_{i}\left(C_{i}^{\prime}\right) \leq E_{\pi}\left(U_{i}\left(C_{i}^{\prime}\right)\right)$. Notice that $v_{i}\left(C_{i}^{\star}\right)=E_{\pi}\left(U_{i}\left(C_{i}^{\star}\right)\right)$. Hence, $v_{i}\left(C_{i}^{\prime}\right) \leq v_{i}\left(C_{i}^{\star}\right)$, and $\left(p^{\star}, C^{\star}\right)$ is an equilibrium of $\mathcal{E}_{4}$.

(ii) Let $\left(p^{\star}, C^{\star}\right)$ be an equilibrium of $\mathcal{E}_{4}$. Assume $\nu_{i}$ is convex and $U_{i}$ satisfy $\mathbf{U m}$ for all $i$. Then $C_{i}^{\star} \gg 0$ for all $i$. From proposition 5.2 and the first theorem of welfare, $C_{i}^{j \star}=C_{i}^{j^{\prime} \star}$ for all $j, j^{\prime}$ and all $i$.

From first-order conditions and $\mathbf{U m}$, there exists $\lambda_{i} \in \mathbb{R}_{+}$for all $i$, such that $p^{\star} \in \lambda_{i} \partial v_{i}\left(C_{i}^{\star}, \ldots, C_{i}^{\star}\right)$. Therefore, $p^{\star}=\left(\lambda_{i} \nabla U_{i}\left(C_{i}^{\star}\right) \pi_{i}^{1}, \ldots, \lambda_{i} \nabla U_{i}\left(C_{i}^{\star}\right) \pi_{i}^{k}\right)$ where $\pi_{i} \in \operatorname{core}\left(\nu_{i}\right)$. Summing over $p^{\star}$ 's components, one gets:

$\sum_{j} \lambda_{i} \nabla U_{i}\left(C_{i}^{\star}\right) \pi_{i}^{j}=\sum_{j} p^{j \star}=\sum_{j} \lambda_{i^{\prime}} \nabla U_{i^{\prime}}\left(C_{i^{\prime}}^{\star}\right) \pi_{i^{\prime}}^{j}$ that is: $\lambda_{i} \nabla U_{i}\left(C_{i}^{\star}\right)=\lambda_{i^{\prime}} \nabla U_{i^{\prime}}\left(C_{i^{\prime}}^{\star}\right) \forall i, i^{\prime}$

Hence, $\pi_{i}^{j}$ is independent of $i$ for all $j$, i.e. $\pi_{i} \in \cap_{i} \operatorname{core}\left(\nu_{i}\right)=\mathcal{P}$. Let $q^{\star}=\lambda_{i} \nabla U_{i}\left(C_{i}^{\star}\right)$ and $\pi=\pi_{i}$. One gets $p^{\star}=\left(q^{\star} \pi^{1}, q^{\star} \pi^{2}, \ldots, q^{\star} \pi^{k}\right)$ with 
$\pi \in \mathcal{P}$. It follows from proposition 5.1 that $\left(p^{\star}, C^{\star}\right)$ is an equilibrium of the vNM economy with utility index $U_{i}$ and probability $\pi$.

This proposition suggests equilibrium indeterminacy if $\mathcal{P}$ contains more than one probability distribution. This road is explored further in Tallon [1997] and Dana [1998]. A direct corollary concerns existence:

Corollary $\mathbf{5 . 1}$ Under $\mathbf{C}$, there exists an equilibrium of $\mathcal{E}_{4}$.

Hence, since capacities satisfying assumption $\mathbf{C}$ need not be convex, convexity of the preferences (which is equivalent, in the CEU setup, to the convexity of the capacity and concavity of the utility index, see Chateauneuf and Tallon [1998]) is not necessary to prove that an equilibrium exists in this setup.

Malinvaud [1973] noticed that P.O. allocations could be decentralized through insurance contract in a large economy. Cass, Chichilnisky and Wu [1996] showed, in an expected utility framework, how this decentralization can be done in a finite economy: agents of the same type share their risk through (actuarially fair) mutual insurance contract. The same type of argument could be used here in the Choquet expected utility case. It is an open issue whether P.O. allocations can be decentralized with mutual insurance contract where agents in the same pool have different capacities. 


\section{References}

A. Billot, A. Chateauneuf, I. Gilboa and J.-M. Tallon. Sharing beliefs: between agreeing and disagreeing. Cahiers Eco\&Maths 98.30, Université Paris I, 1998. Forthcoming Econometrica.

K. Borch. Equilibrium in a reinsurance market. Econometrica, 30:424-444, 1962.

D. Cass, G. Chichilnisky and H.-M. Wu. Individual risk and mutual insurance. Econometrica, 64(2):333-341, 1996.

A. Chateauneuf and J.-M. Tallon. Diversification, convex preferences and non-empty core. Cahiers Eco\&Maths 98-32, Université Paris I, 1998.

A. Chateauneuf, M. Cohen And R. Kast. A review of some results related to comonotonicity. Cahiers Eco \& Maths 97.32, Université Paris I, 1997.

S. Chew, E. Karni and Z. Safra. Risk aversion in the theory of expected utility with rank dependent preferences. Journal of Economic Theory, 42:370-381, 1987.

R.A. DanA. Pricing rules when agents have non-additive expected utility and homogeneous expectations. Cahier du Ceremade, Université Paris IX, 1998.

D. Denneberg. Non-additive measures and integral. Kluwer Academic Publishers, Dordrecht, 1994.

J. Dow And S. Werlang. Uncertainty aversion, risk aversion, and the optimal choice of portfolio. Econometrica, 60(1):197-204, 1992.

L. Eeckhoudt And C. Gollier. Risk, evaluation, management and sharing. Harvester-Wheatsheaf, 1995.

L. Epstein And T. Wang. Intertemporal asset pricing under Knightian uncertainty. Econometrica, 62(3):283-322, 1994.

P. Ghirardato. Coping with ignorance: unforeseen contingencies and nonadditive uncertainty. University of California at Berkeley, 1994.

E. KARni And D. Schmeidler. Utility theory with uncertainty. In W. Hildenbrand and H. Sonnenschein, editor, Handbook of Mathematical Economics, chapter 33, pages 1763-1831, North-Holland, 1991.

M. Landsberger And I. Meilijson. Co-monotone allocations, BickelLehmann dispersion and the Arrow-Pratt measure of risk aversion. Annals of Operations Research, 52:97-106, 1994. 
M. Magill And M. Quinzir. Theory of incomplete markets. Volume 1, MIT Press, 1996.

E. Malinvaud. The allocation of individual risks in large markets. Journal of Economic Theory, 4:312-328, 1972.

E. Malinvaud. Markets for an exchange economy with individual risks. Econometrica, 41:383-410, 1973.

J. Mossin. Aspects of rational insurance purchasing. Journal of Political Economy, 76:553-568, 1968.

S. MukerJi. Understanding the nonadditive probability decision model. Economic Theory, 9(1):23-46, 1997.

J. Quigain. A theory of anticipated utility. Journal of Economic Behavior and Organization, 3:323-343, 1982.

D. Schmeidler. Integral representation without additivity. Proceedings of the American Mathematical Society, 97(2):255-261, 1986.

D. Schmeidler. Subjective probability and expected utility without additivity. Econometrica, 57(3):571-587, 1989.

J.-M. TALLON. Risque microéconomique, aversion à l'incertitude et indétermination de l'équilibre. Annales d'Economie et de Statistique, 48:211-226, 1997.

J.-M. TAllon. Do sunspots matter when agents are Choquet-expectedutility maximizers? Journal of Economic Dynamics and Control, 22:357368, 1998. 